\title{
Methane-derived carbon flow through host-virus trophic networks in soil
}

Sungeun Lee ${ }^{1}$, Ella T. Sieradzki², Alexa M. Nicolas ${ }^{3}$, Robin L. Walker ${ }^{4}$, Mary K.

4 Firestone $^{2,5}$, Christina Hazard ${ }^{1^{*}}$ and Graeme W. Nicol ${ }^{1^{* \#}}$

$6{ }^{1}$ Environmental Microbial Genomics, Laboratoire Ampère, École Centrale de Lyon, CNRS UMR 5005, Université de Lyon, Ecully 69134, France

82 Department of Environmental Science, Policy and Management, University of California, Berkeley, Berkeley, CA 94720, USA

$10{ }^{3}$ Department of Plant \& Microbial Biology, University of California, Berkeley, Berkeley, CA 94720, USA

$12{ }^{4}$ Scotland's Rural College, Craibstone Estate, Aberdeen, AB21 9YA, United Kingdom

${ }^{5}$ Earth Sciences Division, Lawrence Berkeley National Laboratory, Berkeley, CA

1494720, USA

*Joint senior author, " Corresponding author

\section{Abstract}

18 The concentration of atmospheric methane continues to increase with microbial communities controlling soil-atmosphere fluxes. While there is substantial knowledge

20 of the diversity and function of organisms regulating methane production and consumption, the frequency and impact of interactions with viruses on their activity in

22 soil is unknown. Metagenomic sequencing of soil microbial communities has enabled identification of linkages between viruses and hosts. However, determining host-virus

24 linkages through sequencing does not determine whether a virus or a host are active. In this study, we identified active individual interactions in situ by following the transfer

26 of assimilated carbon from active hosts to viruses. Using DNA stable-isotope probing combined with metagenomic analyses, we characterized methane-fueled microbial networks in acidic and neutral pH soils, specifically primary and secondary utilisers of carbon, together with the recent transfer of methane-derived carbon to viruses. Sixty-

30 three percent of viral contigs from replicated soil incubations contained genes associated with known methanotrophic bacteria. Genomic sequences from ${ }^{13} \mathrm{C}$ -

32 enriched viruses were present in clustered regularly interspaced short palindromic repeats (CRISPR) arrays of multiple, closely-related Methylocystis populations, 
34 revealing differences in their history of viral interaction. Viruses infecting nonmethanotrophic methylotrophs and heterotrophic predatory bacteria were also identified through the analysis of shared homologous genes, demonstrating that carbon is transferred to a diverse range of viruses associated with methane-fueled microbial food networks.

\section{$40 \quad$ Main text}

Microorganisms play a central role in global carbon (C) biogeochemical cycling in soil

systems. Soil is one of the most diverse habitats in the biosphere and can typically contain $10^{9}-10^{10}$ prokaryotic cells (Frossard et al., 2016) or viruses (Williamson et al.,

44 2017) per g. Infection by viruses facilitates the horizontal transfer of genes and viral lysis acts as a control of host abundance and releases nutrients. In the marine environment, $20-40 \%$ of prokaryotes are lysed on a daily basis with the release of 150 Gt of carbon annually (Suttle, 2007). However, the role of viruses in influencing prokaryotic ecology in soil remains comparatively unknown (Emerson, 2019). In particular, difficulties remain in identifying the frequency of active interactions between native host and virus populations in situ, largely due to a lack of tools to study interactions within the highly complex and heterogeneous soil environment. While redqueen or 'arms race' dynamics have not yet been observed in natural soil populations as they have in marine systems (Ignacio-Espinoza et al., 2020), studies have shown viruses can coevolve with their hosts in soil and that hosts change in their susceptibility to infection (Gómez and Buckling, 2011). Shotgun sequencing of diverse soil microbial communities has enabled identification of linkages between viruses and hosts involved in carbon cycling both through identifying CRISPR spacer sequences in viral genomes and the presence of viral genes encoding enzymes involved in complex carbon degradation (Emerson et al., 2018). However, determining virus-host associations in situ with these methods does not elucidate the frequency of viral infections, with linkages potentially associated with populations not active under current conditions, or even relic DNA (Carini et al., 2017).

Methanotrophs are a critically important group in soil systems, removing $5 \%$ of atmospheric methane (Curry, 2007) and controlling fluxes to the atmosphere from methanogenic activity in anoxic compartments (Le Mer and Roger, 2001). Aerobic methanotrophs use $\mathrm{CH}_{4}$ for both carbon and energy requirements and key representatives in soil belong to the type I Gammaproteobacteria family 
68 Methylococcaceae, type II Alphaproteobacteria families Methylocystaceae and Beijerinckiaceae, and Methylacidiphilaceae of the Verrucomicrobia (Kneif, 2015). Soil

$70 \mathrm{pH}$ is one of many factors influencing methanotroph activity, with type I and type II methanotrophs often dominating activity in neutral and acidic $\mathrm{pH}$ soils, respectively

72 (Zhao et al., 2020). In addition, a wide variety of non-methanotrophic methylotrophs utilise methanol produced and excreted by methanotrophs, and together

74 methanotrophic and other methylotrophic single carbon compound (C1)-utilising consortia assimilate methane-derived carbon in a variety of habitats (Chistoserdova 76 et al., 2010).

A widely used technique for identifying active populations within a diverse 78 microbial community in environmental samples, including methanotrophs, is DNA stable isotope probing (SIP) (Radajewski et al., 2002). Incorporation of a substrate 80 with an enriched isotope can be traced in genomes of community members, demonstrating utilisation of a specific substrate linked to the associated functional 82 process. As viruses are entirely composed of elements derived from a host cell, their production inside active hosts incorporating an isotopically-enriched substrate will also result in detectable viral isotopic enrichment (Pasulka et al., 2018). In this study we aimed to identify active virus-host interactions within a complex soil habitat by focussing on a taxonomically and functionally restricted group of organisms. By following ${ }^{13} \mathrm{C}$ flow in situ, we aimed specifically to identify lytic DNA viruses of methanotrophs actively using $\mathrm{CH}_{4}$-derived $\mathrm{C}$, including the identification of individual virus-host interactions, and potentially those actively infecting secondary utilisers such as non-methanotrophic methylotrophs.

After aerobically incubating $\mathrm{pH} 4.5$ and 7.5 soils in the presence of ${ }^{12} \mathrm{C}$ - or ${ }^{13} \mathrm{C}$ $92 \mathrm{CH}_{4}$, high buoyant density genomic DNA $\left(>1.732 \mathrm{~g} \mathrm{ml}^{-1}\right)$ containing ${ }^{13} \mathrm{C}$-enriched or ${ }^{12} \mathrm{C}$-high GC mol\% genomic DNA was recovered from triplicate incubations per isotope 94 and soil via isopycnic centrifugation in $\mathrm{CsCl}$ gradients (Supplementary Fig. 1). Six metagenomes were produced from ${ }^{13} \mathrm{C}$ isotopically-enriched DNA samples only (three $\mathrm{pH} 4.5$, three $\mathrm{pH}$ 7.5; Supplementary Table 1). Concentrations of high buoyant density genomic DNA from ${ }^{12} \mathrm{C}_{-} \mathrm{CH}_{4}$ incubations were too low for comparable shotgun sequencing. While this indicated minimal recovery of unenriched DNA in ${ }^{13} \mathrm{C}$ incubated samples, analysis of 16S rRNA gene amplicon libraries prepared from high 100 buoyant density DNA of both ${ }^{12} \mathrm{C}$ and ${ }^{13} \mathrm{C}-\mathrm{CH}_{4}$ incubations confirmed ${ }^{13} \mathrm{C}$-enrichment of C1-utilising populations (Supplementary Text, Supplementary Fig. 2). 
Reads from individual metagenomes were assembled before taxonomic assignment of individual contigs. Reproducibly distinct communities were enriched in 104 the two soils (Supplementary Fig. 2), with six bacterial families representing annotated contigs $>5 \mathrm{kbp}$ to which $>1 \%$ of reads were mapped and all including known $\mathrm{C} 1$ -

106 utilising taxa (Beijerinckiaceae, Bradyrhizobiaceae, Hyphomicrobiaceae, Methylococcaceae, Methylocystaceae and Methylophilaceae). We resolved twenty108 three medium and high-quality (Bowers et al., 2017) metagenome-assembled genomes (MAGs) (Supplementary Table 2), including 12 methanotrophs. Specifically,

1103 MAGs represented gammaproteobacterial type I methanotrophs (Methylobacter) and 9 MAGs represented alphaproteobacterial type II methanotrophs (Methylocystis,

112 Methylosinus or Methylocapsa). Secondary utilisers of methane-derived organic carbon were also identified with 9 MAGs associated with established or putative non-

114 methanotrophic methylotrophs, lacking methane oxidation machinery but capable of utilising methanotroph-derived methanol (see Supplementary Text). These included

116 representatives of the Gemmatimonadales, Hyphomicrobium, Herminiimonas and Rudaea, the latter two, to our knowledge, not having been previously associated with 118 methylotrophy but possessed predicted methanol and formate dehydrogenases (Supplementary Table 2). Two MAGs represented strains of Bdellovibrio and 120 Myxococcus, known predatory bacteria, indicating that growing methylotrophic populations were preyed upon (Pérez et al., 2018).

$122 \quad$ Lytic virus populations linked to C1-hosts were analysed using metagenome viral contigs (mVCs), predicted using established tools. Using contigs $>10 \mathrm{kbp}$ (Roux 124 et al., 2019) VirSorter (Roux et al., 2015) predicted 270 metagenome viral contigs (mVCs) with a further 4 'likely' mVCs predicted uniquely by DeepVirFinder (Ren et al.,

126 2020) (see Supplementary Text), together representing 227 viral operational taxonomic units (vOTUs) (Paez-Espino et al., 2017). Analysis of the normalised read 128 mapping for mVCs demonstrated that, as with the bacterial communities, active ${ }^{13} \mathrm{C}$ enriched viral populations were reproducibly distinct between acidic and neutral $\mathrm{pH}$ 130 soils (Supplementary Fig. 3).

mVCs were linked to host bacteria using three different approaches: identifying 132 incorporation of viral DNA into spacers of bacterial CRISPR arrays, similarity of homologous genes possessed by both host and virus contigs, and $k$-mer similarity 134 between potential host and virus contigs, the last approach being considered only partially successful (see Supplementary Text). CRISPR arrays were identified in 3 of 
13623 MAGs, each associated with the genus Methylocystis or Methylosinus of the Methylocystaceae (Fig. 1). In the acidic soil, complete CRISPR arrays of growing 138 methanotrophs were associated with two Methylocystis MAGs (MAG identifiers 5 and 6) sharing $79.2 \%$ average nucleotide identity (ANI) and likely representing different 140 species (Jain et al., 2018). A further six CRISPR arrays were identified in unbinned bacterial contigs all possessing the same direct repeat (DR) sequence. These eight 142 arrays varied in size ranging from 9 to 114 DRs and contained a total of 432 spacers, and were in the same size range of Methylocystaceae CRISPR arrays from sequenced

144 genomes (Supplementary Table 3). Comparison of spacer incorporation between arrays revealed that these multiple closely-related populations had different histories

146 of viral interaction and subsequent spacer incorporation. Genome sequences from ${ }^{13} \mathrm{C}$-enriched viral populations were represented by seven mVCs and matched $29.5 \%$

148 of spacers. In addition, $7.9 \%$ of spacers possessed a one nucleotide mismatch, all of which represented a synonymous substitution, indicating that variation was the result

150 of mutations in viral genomes increasing their ability to evade CRISPR-CAS defense systems or genetic variation in closely related viral populations. Only three pairs of spacers were identical, with each pair member located on a different array. Variation in virus host range was also observed, with 3 and 2 mVCs linked to only one or both

154 Methylocystis MAGs, respectively.

Surprisingly, a large number of spacers in individual Methylocystis CRISPR 156 arrays were linked to the same virus, with up to 31 being homologous to protospacer sequences in one mVC. To provide support that these multiple spacers were derived 158 from Methylocystis-associated viruses, mVCs were examined for host-specific conserved protospacer-adjacent motif (PAM) sequences (Mojica et al., 2009).

160 Consistent with the identification of genuine protospacers, 138 of linked 146 spacers (i.e. all possessing $\leq 1$ mismatch) had the conserved PAM sequence 'TTC' (target162 centric orientation) (Leenay and Beisel, 2017). The variation in spacer position between the arrays revealed temporal differences in virus infections. For example, the most recently integrated spacer in 3 of 8 different Methylocystis CRISPRs was derived from a ${ }^{13} \mathrm{C}$-enriched virus represented by $\mathrm{mVC}$ _12213_cat2, suggesting the possibility of incorporation occurring during the incubation of the experiment.

Further analysis of all CRISPR arrays (i.e. including those in unbinned contigs) 168 linked to ${ }^{13} \mathrm{C}$-enriched viruses revealed that the majority of viruses were associated with methanotrophic populations (Fig. 2a). In total, 11 different variants were identified 
170 (i.e. each having a unique DR sequence) with 9 linked to Methylocystaceae or Methylococcaceae populations. DR sequences generally possessed high sequence

172 similarity to those in CRISPR arrays from cultivated strain genomes of the same family, although only CRISPR array 6 had a DR sequence that was identical (Supplementary

174 Table 3). Individual DR variants were restricted to either $\mathrm{pH} 4.5$ or 7.5 soil. Using $100 \%$ sequence identity in searches between CRISPR spacer and mVC protospacer

176 sequences, 19 VirSorter-predicted mVCs were linked to all CRISPR array variants. In addition, analysis of shorter mVCs ranging 5-10 kbp identified two additional linked 178 mVCs (mVC_08964_cat.3 (9.8 kbp) and mVC_28139_DVF (5.1 kbp)). One third of CRISPR linked-mVCs were categorized at the lowest level of confidence (i.e.

180 category-3 by VirSorter (Roux et al., 2015) or 'possible' by DeepVirFinder (Ren et al., 2020), suggesting that retaining only higher confidence contigs may exclude a

182 substantial proportion of bona fide methanotroph virus-derived contigs in uncharacterised environments such as soil.

184 Analysis of tetranucleotide frequencies (TETRA) (Wang et al., 2017) clustered the 21 mVCs into three groups that were associated with the Methylocystaceae, Methylococcoceae and an unknown group (Fig. 2b). The majority of viruses infected members of the Methylocystaceae family, with those infecting populations of the

188 Methylocystis and Methylosinus genera restricted to acidic and neutral pH soils, respectively. TETRA correlation coefficients of all Methylocystaceae-linked viruses

190 were in the same range both within and between either genus, suggesting co-evolution with their host rather than genetic drift and divergence was the primary mechanism for defining specific associations with Methylocystis or Methylosinus strains.

Identification of homologous genes in mVCs that were shared with prokaryotic 194 genomes were always consistent with host-virus linkages established using spacer sequences from MAG CRISPR arrays. Specifically, BLASTp searches of genes 196 present in the $9 \mathrm{mVCs}$ linked to Methylocystaceae MAGs via CRISPR spacer sequences all contained 'best hits' (identity $>30 \%$, e-value $<10^{-5}$, bit score $>50$ and query cover $>70 \%$ ) to a minimum of 5 homologues also found in Methylocystaceae genomes. This was therefore used as a criterion for establishing host-virus linkages.

200 Sixty-three percent of mVCs contained a homologue that was linked to genomes of known C1-utilising bacteria, with $35 \%$ linked specifically to populations from the 202 Methylocystaceae, Methylococcaceae or Hyphomicrobiaceae (Fig. 3a). While analysis of bacterial homologues in mVCs identified the taxonomic family of the 
204 assumed dominant host, they also indicated that individual viruses may infect hosts of other families of the same taxonomic order, including those at other trophic levels.

206 Specifically, within the Rhizobiales, mVCs linked to Methylocystaceae also contained homologues shared with Bradyrhizobiaceae, Methylobacteriaceae and Rhizobiaceae

208 (Fig. 3b), indicating that viruses of methanotrophs may also infect non-methanotrophic methylotrophs that are active at the same time.

$210 \mathrm{CH}_{4}$-derived $\mathrm{C}$ was also transferred to viruses of secondary or tertiary utilisers. One group of mVCs were linked to methylotrophic Hyphomicrobiaceae and a second

212 to a phylogenetically diverse range of nitrogen-fixing Rhizobia i.e. Bradyrhizobiaceae, Phyllobacteriaceae and Rhizobiaceae. These lineages contain known methylotrophs,

214 methanol dehydrogenases have been identified in a range of rhizobial species (Huang et al., 2019) and these mVCs also contained homologues found in the genomes of

216 nodulating Methylobacterium strains (Green and Ardley, 2018). Viruses of predatory Bdellovibrio and Myxococcales bacteria were predicted, consistent with the recovery 218 of corresponding bacterial MAGs in ${ }^{13} \mathrm{C}$-enriched DNA. One mVC (20210-cat_2) was linked to the genus Bdellovibrio (sharing 11 of $67 \mathrm{mVC}$ genes) and three category-3 220 mVCs (i.e. possible viruses) was linked to Myxococcales populations, containing gene homologous to four families within the order. The high isotopic labelling of both

222 heterotrophic predators (with no identifiable C1-utilising capability) and their viruses indicate that the predators were feeding primarily on $\mathrm{C} 1$-utilisers, as carbon 224 incorporated by feeding on unlabelled bacteria would dilute the enrichment in predators. As such, it also indicates that predatory bacteria may have preference for preying upon growing populations rather than the non-C1-utilising majority.

Gene-sharing network analysis of mVCs with viruses in the NCBI RefSeq 228 database and other metagenome studies were analysed using vConTACT 2.0 (Jang et al., 2019). Any linkages with RefSeq viruses typically had low scores (i.e. sharing a

230 low number of homologues) and were linked to viruses of hosts that were inconsistent with our homologue-based predictions (Supplementary Table 4). No linkages were 232 observed with recently reported giant viruses of methanotrophs in freshwater lakes (Chen et al., 2020). However, in a recent study of 197 metagenomes from Swedish 234 peatland soil, Emerson et al. (2018) identified 13 viruses linked to methanotrophs. Intriguingly, 8 of these were linked in our viral gene-sharing network, with both studies 236 predicting Methylocystaceae hosts using different methods of host annotation (Supplementary Text, Supplementary Fig. 5) and revealing the distribution of specific 
238 Methylocystaceae-associated viral groups present in different geographical areas and soil types. Analysis of gene-sharing networks of mVCs from this study indicated that 240 there were two distinct Methylocystaceae-linked viral clusters which also varied in their distribution in both soils. Specifically, one cluster was associated with low pH only

242 whereas the second cluster contained viruses found in both $\mathrm{pH} 4.5$ and 7.5 soils, including those linked by CRISPR spacer sequences. Individual networks of

244 Methylococcaceae- and rhizobia-associated mVCs were also identified, typically associated with one of the two soils of contrasting $\mathrm{pH}$. Taxonomically-linked mVCs

246 with $\geq 5$ homologues were consistently placed in networks with other mVCs containing 1-4 homologues from the same methylotrophic families, confirming host linkage to a 248 larger number of mVCs.

mVCs contained 8,174 genes, with 49.6\% (4,054) annotated representing 606

250 unique functions. Of these, genes encoding viral proteins accounted for $9.8 \%$ (397 genes) and included major capsid proteins, tail proteins, integrases, portal proteins

252 and terminases. Bacterial proteins used for viral replication accounted for 5.1\% (206 genes). A number of metagenomic studies have demonstrated that viruses can

254 possess genes encoding sub-unit $\mathrm{C}$ of ammonia or particulate methane monooxygenases as auxiliary metabolic genes (AMGs) (Chen et al., 2020; Ahlgren et

256 al., 2019) which are also typically found as isolated genes in genomes in addition to being present in clusters or operons encoding A and B sub-units (Nicol and Schleper, 258 2006). In this study, one low confidence mVC (7.3 kbp, category-3) was identified as containing an isolated $p m o C$ gene that was phylogenetically related to growing 260 Methylocystis populations but was distinct from $p m o C$ sequences found in viruses associated with freshwater Methylocystis populations (Chen et al., 2020)

262 (Supplementary Fig. 6).

In summary, these results demonstrate that by following carbon flow, viruses 264 and hosts associated with a critical biogeochemical process can be identified at the scale of individual populations, and currently active interactions at different trophic 266 levels examined within the highly complex soil environment. Type I and II methanotrophs interact with evolutionarily distinct groups of viruses and the 268 composition of CRISPR arrays of Methylocystaceae reveal that they have a continual dynamic interaction with individual viruses. Analysis of shared homologues in 
270 individual viral genomes show that they may interact with host populations at different trophic levels within a methane-fuelled network.

272

\section{Methods}

\section{Soil microcosms}

Triplicate soil samples were collected in February 2018 at $1 \mathrm{~m}$ intervals from the upper

$27610 \mathrm{~cm}$ of $\mathrm{pH} 4.5$ and 7.5 soil sub-plots of a pH gradient maintained since 1961 and under an 8-year crop rotation (SRUC, Craibstone Estate, Aberdeen, Scotland; UK grid reference NJ872104) (Kemp et al., 1992). The crop at the time of sampling was potatoes. Soil (podzol, sandy-loam texture) was sieved (2 $\mathrm{mm}$ mesh size) and

280 microcosms established in triplicate for each soil $\mathrm{pH}$ and isotope in $144 \mathrm{ml}$ serum bottles containing $14.30 \mathrm{~g}$ soil (10 g dry weight equivalent) with a $30 \%$ volumetric water

282 content, equivalent to $\sim 60 \%$ water-filled pore space. Bottles were capped and established with a $10 \%$ (v/v) ${ }^{12} \mathrm{C}-\mathrm{CH}_{4}$ or ${ }^{13} \mathrm{C}_{-} \mathrm{CH}_{4}$ (Sigma-Aldrich) headspace $(99 \%$

284 atom enriched), re-opening every 10 days to maintain aerobic conditions before sealing and re-establishing $\mathrm{CH}_{4}$ headspace concentrations. Microcosms were incubated at $25^{\circ} \mathrm{C}$ and destructively sampled after 30 days with soil archived immediately at $-20^{\circ} \mathrm{C}$.

\section{DNA-SIP}

290 Genomic DNA was extracted from $0.5 \mathrm{~g}$ soil samples using a CTAB buffer phenol:chloroform: isoamyl alcohol bead-beating protocol and subjected to isopycnic centrifugation in $\mathrm{CsCl}$ gradients, recovery and purification as previously described (Nicol and Prosser, 2011). Briefly, 6 ug of genomic DNA was added to $8 \mathrm{ml} \mathrm{CsCl-Tris}$

294 EDTA solution (refractive index (RI) of 1.4010; buoyant density of $1.71 \mathrm{~g} \mathrm{ml}^{-1}$ ) in polyallomer tubes before sealing and ultracentrifugation at 152,000 × $g(50,000 \mathrm{rpm})$ 296 in a MLN80 rotor (Beckman-Coulter) for $72 \mathrm{~h}$ at $25^{\circ} \mathrm{C}$. CsCl gradients were fractionated into 350 ul aliquots using an in-house semi-automated fraction recovery system before determining RI and recovering DNA. The relative abundance of bacterial $16 \mathrm{~S}$ rRNA and methanotrophic pmoA genes in genomic DNA distributed across the $\mathrm{CsCl}$

300 gradients was determined by qPCR in a Corbett Rotor-Gene 6000 thermocycler (Qiagen) using primer sets P1(341f)/P2(534r) (Muyzer et al., 1993) and A189F/A682R

302 (Holmes et al., 1995) respectively. Twenty-five $\mu$ l reactions contained $12.5 \mu \mathrm{L} 2 \mathrm{X}$ QuantiFast SYBR Green Mix (Qiagen), $1 \mu \mathrm{M}$ of each primer, $100 \mathrm{ng}$ of T4 gene protein 
30432 (Thermo Fisher), $2 \mu$ l of standard $\left(10^{8}-10^{2}\right.$ copies of an amplicon-derived standard) or 1/10 diluted DNA. Thermocycling conditions consisted of an initial denaturation step

306 of $15 \mathrm{~min}$ at $95^{\circ} \mathrm{C}$ for both assays followed by 30 cycles of $15 \mathrm{~s}$ at $94^{\circ} \mathrm{C}, 30 \mathrm{~s}$ at $60^{\circ} \mathrm{C}$, $30 \mathrm{~s}$ at $72^{\circ} \mathrm{C}$ for the $16 \mathrm{~S}$ rRNA gene assay or $60 \mathrm{~s}$ at $94^{\circ} \mathrm{C}, 60 \mathrm{~s}$ at $56^{\circ} \mathrm{C}, 60 \mathrm{~s}$ at $72^{\circ} \mathrm{C}$

308 for the pmoA gene assay, followed by melt-curve analysis. All assays had an efficiency between $93-97 \%$ with an $r^{2}$ value $>0.99$. Genomic DNA from four fractions with a

310 buoyant density $>1.732 \mathrm{~g} \mathrm{ml}^{-1}$ were then pooled for each ${ }^{12} \mathrm{C}$ - and ${ }^{13} \mathrm{C}$ - $\mathrm{CH}_{4}$-incubated replicate for $16 \mathrm{~S}$ rRNA gene amplicon sequence and metagenomic analysis.

312

Metagenome sequencing, assembly \& annotation

314 Library preparation and sequencing was performed at the Joint genome Institute (JGI), Berkeley, USA. Libraries were produced from fragmented DNA using KAPA

316 Biosystems Library Preparation Kits (Roche) and quantified using KAPA Biosystems NGS library qPCR kits. Indexed samples were sequenced ( 2 × 150 bp) on the Illumina

318 NovaSeq platform with NovaSeq XP v1 reagent kits and a S4 flowcell. Raw reads were processed with JGl's RQCFilter2 pipeline that utilised BBTools v38.51 (Bushnell,

320 2016). Reads containing adapter sequences were trimmed and those with $\geq 3 \mathrm{~N}$ bases or $\leq 51$ bp or $\leq 33 \%$ of full-read length were removed along with PhiX sequences using

322 BBDuk, and reads mapped to human, cat, dog or mouse references at 95\% identity were removed using BBMap. De novo contig assembly of the 100 - 196 million quality-

324 controlled reads per metagenome was performed using MetaSPAdes v3.13.0 (Nurk et al., 2016). The 1 - 2 million contigs per metagenome were then concatenated 326 together, and contigs larger than $5 \mathrm{kbp}$ were dereplicated at $99 \%$ average nucleotide identity (ANI) using PSI-CD-HIT v4.6.1 (Fu et al., 2012) and binned using MetaWRAP 328 v1.2.1 (Uritskiy et al., 2018) (Supplementary Table 5). Bin completion and contamination was determined by CheckM v1.0.12 (Parks et al., 2015). Taxonomic 330 annotation of contigs was performed using Kaiju (Menzel et al., 2016) with the NCBI RefSeq database (Release 94; 25 June 2019) (O'Leary et al., 2016) and MAGs using 332 GTDB-Tk v0.3.2 (Chaumeil et al., 2019) with the Genome Taxonomy Database (release 89, 21 June 2019) (Parks et al., 2018). Protein sequence annotation was 334 performed using InterProScan 5 (e-value <10-5) (Jones et al., 2015). Pairwise ANI comparison of MAGs was calculated using FastANI (Jain et al., 2019). 
338 16S rRNA genes were amplified using primers 515F/806R (Walters et al., 2015) followed by library preparation and sequencing on an Illumina MiSeq sequencer as 340 previously described (Finn et al., 2020). Reads with a quality score $<20$ and length < 100 bp were discarded using FASTX-Toolkit v0.0.13

342 (http://hannonlab.cshl.edu/fastx_toolkit/). High-quality reads were merged using PANDAseq v2.11 (Masella et al., 2012), and denoising and chimera removal 344 performed with UNOISE3 (Edgar, 2016). Amplicon sequence variants (ASVs) were annotated using the RDP classifier v2.11 (Wang et al, 2007). Non-metric 346 multidimensional scaling of Bray-Curtis dissimilarity derived from the relative abundance of ASVs was performed with the metaMDS function in the vegan package

348 (Oksanen et al., 2019) in R v3.6.0.

\section{$350 \quad$ Virus prediction}

Metagenomic viral contigs (mVCs) were predicted from 9,190 contigs $>10 \mathrm{kbp}$ using

352 VirSorter (Roux et al., 2015), retaining non-prophage category-1, -2 or -3 mVCs, representing "most confident", "likely" and "possible". DeepVirFinder (Ren et al., 2020)

354 was also used to predict mVCs from contigs $>10 \mathrm{kbp}$, with those with a $p$-value $<0.05$ and a score $\geq 0.9$ or $\geq 0.7$, representing "confident" and "possible", respectively 356 (Supplementary Table 6). The relative abundance of each $\mathrm{mVC}$ in the six metagenomes was determined using the MetaWRAP-Quant_bins module (Uritskiy et 358 al., 2018) and a heatmap produced using the heatmaply package in R v3.6.0.

Virus-host linkage

CRISPR arrays within MAGs and unbinned contigs were identified using the CRISPR 362 Recognition Tool v1.2 (Bland et al., 2007) (Supplementary Table 7). DR and spacer sequences were extracted before performing 100\% identity searches against positive

364 and negative strands to identify MAGs or contigs with direct repeats and the viral origin of spacers using Seqkit commands (Shen et al., 2016). After identification of matched spacer sequences in mVCs, 10 nucleotides before and after the spacer sequence were extracted to identify associated host-specific PAM sequences. Conserved and variant PAM sequences were manually identified. Correlation coefficients of pairwise comparison of the tetra-nucleotide frequencies (TETRA) between unique CRISPR-

370 associated mVCs were calculated using Python package pyani v0.2.10 (Pritchard et al., 2016). To identify homologous genes shared between CRISPR-linked viruses and 
372 hosts, gene prediction was performed using Prodigal v2.6.3 (Hyatt et al., 2010) with the -p meta option followed by protein alignment with Blastp (identity $>30 \%$, e-value <

$37410^{-5}$, bit score $>50$ and query cover $>70 \%$ ) and protein sequence annotation using InterProScan $5\left(\right.$ e-value $\left.<10^{-5}\right)$. Gene homology between all mVCs and prokaryotes in

376 the NCBI nr database was determined using Diamond Blastp (e-value $<10^{-5}$ ) (Buchfink et al., 2015). Virus-host prediction using k-mer frequencies was performed with WIsH

378 v1.0 (p-values <0.05) (Galiez et al., 2017). Networks based on shared gene content was constructed using vConTACT 2.0 (Jang et al., 2019) with the NCBI RefSeq 380 database (Release 94; 25 June 2019).

382 Phylogenetic analysis of $\mathrm{PmoC}$ and $\mathrm{PxmC}$ protein sequences

Maximum likelihood analysis of inferred protein sequences of membrane-bound

384 monooxygenase $\mathrm{C}$ sub-units from methanotroph MAGs and reference sequences (Supplementary Table 8) was performed on unambiguously aligned sequences using

386 PhyML 3.0 (Guindon et al., 2010) with automatic model selection (LG substitution, gamma distribution (0.06) and proportion of invariable sites (0.087) estimated).

388 Bootstrap support was calculated from 100 replicates.

\section{Data availability}

Metagenome sequence reads are deposited under NCBI BioProject accession 392 numbers PRJNA621430 - PRJNA621435. Metagenome draft assemblies are accessible through the JGI Genome Portal (DOI: 10.25585/1487501). Amplicon 394 sequence data is deposited in the NCBI Sequence Read Archive with BioProject accession number PRJNA676099.

\section{Acknowledgments}

398 The sequencing data were generated under JGI Community Science Program proposal 503702 awarded to GWN and $\mathrm{CH}$. The work conducted by the U.S.

400 Department of Energy Joint Genome Institute, a DOE Office of Science User Facility, is supported by the Office of Science of the U.S. Department of Energy under Contract

402 No. DE-AC02-05CH11231. This work was funded by an AXA Research Chair awarded to GWN and a France-Berkeley Fund grant (2018-2019) awarded to GWN and MKF.

404 The $\mathrm{pH}$ gradient experiment is funded through the Scottish Government RESAS 2016- 
2021 programme. The authors would like to thank Dr. Joanne Emerson for valuable 406 discussion.

\section{Author contributions}

The research program was conceived by and funded from grants awarded to GWN,

$410 \mathrm{CH}$ and MF. SL, CH and GWN designed the experiment and wrote the manuscript. SL performed experiments and analyses. ES, AN and MF advised on bioinformatic

412 approaches, discussed data and commented on the manuscript. RW coordinated soil sampling and commented on the manuscript. All authors approved the manuscript.

414

\section{References}

416

Ahlgren, N.A., Fuchsman, C.A., Rocap, G. \& Fuhrman, J.A. Discovery of several

418 novel, widespread, and ecologically distinct marine Thaumarchaeota viruses that encode amoC nitrification genes. ISME J. 13, 618-631 (2019).

420 Angel, R., Claus, P., \& Conrad, R. Methanogenic archaea are globally ubiquitous in aerated soils and become active under wet anoxic conditions. ISME J. 6, 847-862 422 (2012).

Bland, C. et al. CRISPR Recognition Tool (CRT): a tool for automatic detection of

424 clustered regularly interspaced palindromic repeats. BMC Bioinform. 8, 209 (2007).

Bowers, R.M. et al. Minimum information about a single amplified genome (MISAG) 426 and a metagenome-assembled genome (MIMAG) of bacteria and archaea. Nat. Biotech. 35, 725-731 (2017).

428 Buchfink, B., Xie, C. \& Huson, D.H. Fast and sensitive protein alignment using DIAMOND. Nat. Methods 12, 59-60 (2015).

430 Bushnell, B. BBTools software package. http://sourceforge net/projects/bbmap (2016).

432 Carini, P., Marsden, P.J., Leff, J.W., Morgan, E.E., Strickland, M.S. \& Fierer N. Relic DNA is abundant in soil and obscures estimates of soil microbial diversity. Nat.

434 Microbiol. 2, 16242 (2017).

Chaumeil, P-A., Mussig, A.J., Hugenholtz, P. \& Parks, D.H. GTDB-Tk: a toolkit to 436 classify genomes with the Genome Taxonomy Database. Bioinformatics 36, 19251927 (2019). 
438 Chen, L.-X. et al. Large freshwater phages with the potential to augment aerobic methane oxidation. Nat. Microbiol. In press (2020).

440 Chistoserdova, L., Kalyuzhnaya, M.G. \& Lidstrom, M.E. The expanding world of methylotrophic metabolism. Annu. Rev. Microbiol. 63, 477-499 (2009).

442 Curry, C.L. Modeling the soil consumption of atmospheric methane at the global scale, Global Biogeochem. Cycles 21, GB4012 (2007).

444 Edgar, R.C. UNOISE2: improved error-correction for Illumina 16S and ITS amplicon sequencing. Preprint at https://doi.org/10.1101/081257 (2016).

446 Emerson, J.B. et al. Host-linked soil viral ecology along a permafrost thaw gradient. Nat. Microbiol. 3, 870-880 (2018).

448 Emerson, J.B. Soil viruses: A new hope. mSystems 4, e00120-19 (2019).

Finn, D.R., Lee, S., Lazén, M.B., Nicol, G.W. \& Hazard, C. Cropping systems that 450 improve richness convey greater resistance and resilience to soil fungal, relative to prokaryote, communities. Preprint at https://doi.org/10.1101/2020.03.15.992560 452 (2020).

Frossard, A., Hammes, F., \& Gessner, M.O. Flow cytometric assessment of bacterial 454 abundance in soils, sediments and sludge. Front. Microbiol. 7, 903 (2016).

Fu, L., Niu, B., Zhu, Z., Wu, S. \& Li, W. CD-HIT: accelerated for clustering the next456 generation sequencing data. Bioinformatics 28, 3150-3152 (2012).

Galiez, C, Siebert, M., Enault, F., Vincent, J. \& Söding, J. WisH: who is the host? 458 Predicting prokaryotic hosts from metagenomic phage contigs. Bioinformatics 33, 3113-3114 (2017).

460 Gómez, P. \& Buckling. Bacteria-phage antagonistic coevolution in soil. Science 332, 106-109 (2011).

462 Green, P.N and Ardley, J.K. Review of the genus Methylobacterium and closely related organisms: a proposal that some Methylobacterium species be reclassified into 464 a new genus, Methylorubrum gen. nov. Int. J. Syst. Evol. Microbiol. 68, 2727-2748 (2018).

466 Guindon, S. et al. New algorithms and methods to estimate maximum-likelihood phylogenies: Assessing the Performance of PhyML 3.0. Syst. Biol. 59, 307-21 (2010). 
468 Holmes, A.J., Costello, A., Lidstrom, M.E. \& Murrell, J.C. Evidence that particulate methane monooxygenase and ammonia monooxygenase may be evolutionarily 470 related. FEMS Microbiol. Lett. 132, 203-208 (1995).

Huang, J. et al. Rare earth element alcohol dehydrogenases widely occur among 472 globally distributed, numerically abundant and environmentally important microbes. ISME J. 13, 2005-2017 (2019).

474 Hyatt, D. et al. Prodigal: prokaryotic gene recognition and translation initiation site identification. BMC Bioinform. 11, 119 (2010).

476 Ignacio-Espinoza, J. C., Ahlgren, N.A. \& Fuhrman, J.A. Long-term stability and Red Queen-like strain dynamics in marine viruses. Nat. Microbiol. 5, 265-271 (2020).

478 Jain, C., Rodriguez-R, L.M., Phillippy, A.M. \& Konstantinidis, K.T. High throughput ANI analysis of $90 \mathrm{~K}$ prokaryotic genomes reveals clear species boundaries. Nature Comm.

$480 \quad 9,5114(2018)$.

Jang, H.B. et al. Taxonomic assignment of uncultivated prokaryotic virus genomes is 482 enabled by gene-sharing networks. Nat. Biotechnol. 37, 632-639 (2019).

Jones, P. et al. InterProScan 5: genome-scale protein function classification. 484 Bioinformatics 30, 1236-1240 (2015).

Kemp, J.S., Paterson, E., Gammack, S.M., Cresser, M.S. \& Killham, K. Leaching of 486 genetically modified Pseudomonas fluorescens through organic soils: influence of temperature, soil pH, and roots. Biol. Fert. Soils 13, 218-224 (1992).

488 Kirschke, S., et al. Three decades of global methane sources and sinks. Nat. Geosci. 6, 813-823 (2013).

490 Knief, C. Diversity and habitat preferences of cultivated and uncultivated aerobic methanotrophic bacteria evaluated based on $p m o A$ as molecular marker. Front.

492 Microb. 6, $1346(2015)$.

Le Mer, J. \& Roger, P. Production, oxidation, emission and consumption of methane 494 by soils: a review. Eur. J. Soil Biol. 37, 25-50 (2001).

Leenay, R.T. \& Beisel, C.L. Deciphering, communicating, and engineering the 496 CRISPR PAM. J. Mol. Biol. 429, 177-191 (2017). 
Lyu, Z., Shao, N., Akinyemi, T. \& Whitman, W.B. Methanogenesis. Curr. Biol. 28, 727$498732(2018)$.

Masella, A.P., Bartram, A.k., Truszkowski, J.M., Brown, D.G. \& Neufeld, J.D. 500 PANDAseq: paired-end assembler for illumina sequences. BMC Bioinform. 13, 31 (2012).

502 Menzel, P., Ng, K.L. \& Krogh, A. Fast and sensitive taxonomic classification for metagenomics with Kaiju. Nat. Comm. 7, 11257 (2016).

504 Mojica, F.J.M., Díez-Villaseñor, C., García-Martínez, J., \& Almendros, C. Short motif sequences determine the targets of the prokaryotic CRISPR defence system. Microb.

506 155, 733-740 (2009).

Muyzer, G., De Waal, E.C. \& Uitterlinden, A.G. Profiling of complex microbial 508 populations by denaturing gradient gel electrophoresis analysis of polymerase chain reaction-amplified genes coding for 16 S rRNA. Appl. Environ. Microbiol. 59, 695-700 510 (1993).

Nicol, G.W. \& Prosser, J.I. Strategies to determine diversity, growth and activity of 512 ammonia oxidising archaea in soil. Meth. Enzymol. 496, 3-34 (2011).

Nicol, G.W. \& Schleper C. Ammonia-oxidising Crenarchaeota: important players in the 514 nitrogen cycle? Trends Microbiol. 14, 207-212 (2006).

Nurk, S., Meleshko, D., Korobeynikov, A. \& Pevzner, P.A. metaSPAdes: a new 516 versatile metagenomic assembler. Genome Res. 27, 824-834 (2017).

O'Leary, N.A. et al. Reference sequence (RefSeq) database at NCBI: current status,

518 taxonomic expansion, and functional annotation. Nucleic Acids Res. 44, D733-D745 (2016).

520 Oksanen, J. et al. vegan: Community Ecology Package. https://CRAN.Rproject.org/package=vegan (2019).

522 Paez-Espino, D., Pavlopoulos, G.A., Ivanova, N.N. \& Kyrpides, N.C. Nontargeted virus sequence discovery pipeline and virus clustering for metagenomic data. Nat. Protoc. 12, 1673 (2017). 
Parks, D. H., Imelfort, M., Skennerton, C. T., Hugenholtz, P. \& Tyson, G. W. CheckM: assessing the quality of microbial genomes recovered from isolates, single cells, and metagenomes. Genome Res. 25, 1043-1055 (2015).

528 Parks, D.H. et al. A standardized bacterial taxonomy based on genome phylogeny substantially revises the tree of life. Nat. Biotechnol. 36, 996-1004 (2018).

530 Pérez, J. Moraleda-Muñoz, A., Marcos-Torres, F.J. \& Muñoz-Dorado, J. Bacterial predation: 75 years and counting! Environ. Microbiol. 18, 766-779 (2018).

532 Pratscher, J., Vollmers, J., Wiegand, S., Dumont, M.G., \& Kaster, A.-K. Unravelling the identity, metabolic potential and global biogeography of the atmospheric methaneoxidizing Upland Soil Cluster a. Environ. Microbiol. 20, 1016-1029 (2018).

Pasulka, A.L. et al. Interrogating marine virus-host interactions and elemental transfer 536 with BONCAT and nanoSIMS-based methods. Environ. Microbiol. 20, 671-692 (2018).

Pritchard, L., Glover, R.H., Humphris, S., Elphinstone, J.G. \& Toth, I.K. Genomics and 538 taxonomy in diagnostics for food security: soft-rotting enterobacterial plant pathogens. Anal. Methods 8, 12-24 (2016).

540 Radajewski, S., et al. Identification of active methylotroph populations in an acidic forest soil by stable-isotope probing. Microbiol. 148, 2331-2342 (2002).

542 Ren, J. et al. Identifying viruses from metagenomic data by deep learning. Quant. Biol. 8, 64-77 (2020).

544 Roux, S., Enault, F., Hurwitz, B. L. \& Sullivan, M. B. VirSorter: mining viral signal from microbial genomic data. PeerJ 3, e985 (2015).

546 Roux, S. et al. Minimum information about an uncultivated virus genome (MIUViG). Nat. Biotechnol. 37, 29-37 (2019).

548 Shen, W., Le, S., Li, Y. \& Hu, F. SeqKit: A cross-platform and ultrafast toolkit for FASTA/Q file manipulation. PLoS ONE 11, e0163962 (2016).

550 Suttle, C. A. Marine viruses-major players in the global ecosystem. Nat. Rev. Microbiol. 5, 801-812 (2007).

552 Uritskiy, G.V., DiRuggiero, J. \& Taylor, J. MetaWRAP- a flexible pipeline for genomeresolved metagenomic data analysis. Microbiome 15, 158 (2018). 
554 Walters, W. et al. Improved bacterial 16S rRNA gene (V4 and V4-5) and fungal internal transcribed spacer marker gene primers for microbial community surveys. mSystems

556 1, e00009-15 (2015).

Williamson, K.E., Fuhrmann, J.J., Wommack, K.E., \& Radosevich, M. Viruses in soil

558 ecosystems: an unknown quantity within an unexplored territory. Annu. Rev. Virol. 4, 201-219 (2017).

560 Wang, J. et al. Genomic sequence of 'Candidatus Liberibacter solanacearum' haplotype $\mathrm{C}$ and its comparison with haplotype A and B genomes. PLoS One 12, 562 e0171531 (2017).

Wang, Q., Garrity, G.M., Tiedje, J.M. \& Cole, J.R. Naïve bayesian classifier for rapid

564 assignment of rRNA sequences into the new bacterial taxonomy. Appl. Environ. Microbiol. 73, 5261-5267 (2007).

566 Zhao, J., Cai, Y. \& Jia, Z. The pH-based ecological coherence of active canonical methanotrophs in paddy soils. Biogeosciences 17, 1451-1462 (2020). 
a)

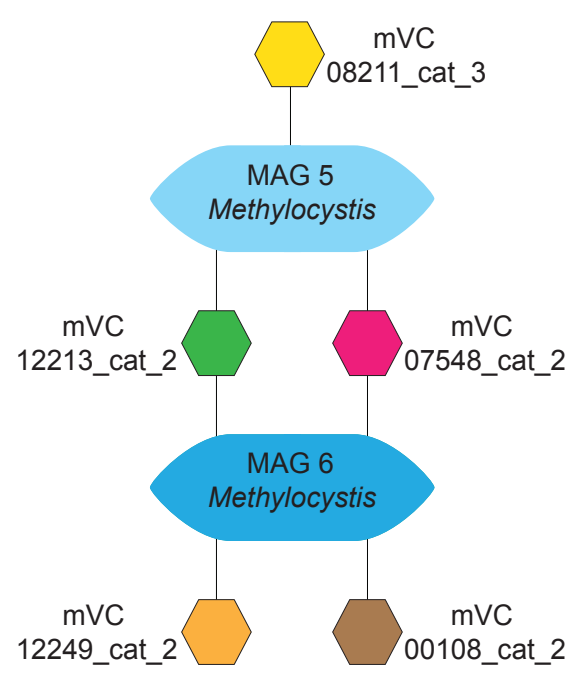

$\mathrm{mVC}$ 00234_cat_2
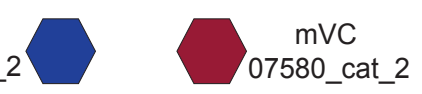

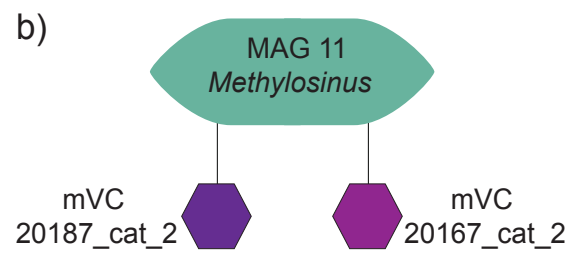

MAG 11 (pH7.5 2 CRISPR 2)

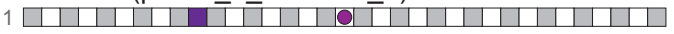

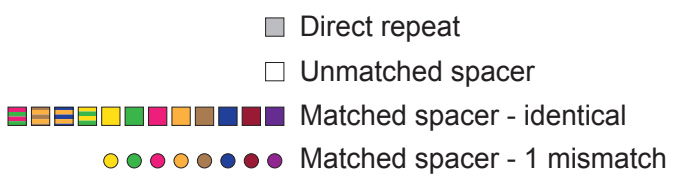

MAG 5 (pH4.5_2_CRISPR_03)

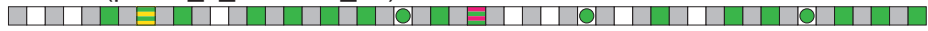

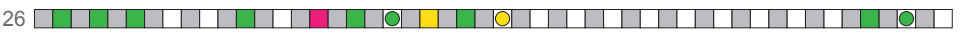

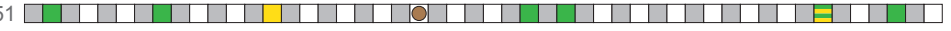

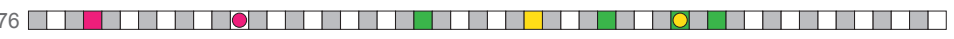

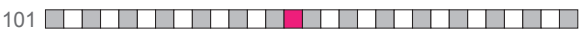

MAG 6 (pH4.5_3_CRISPR_01)

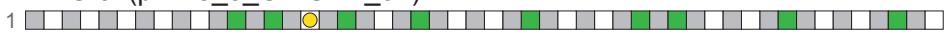

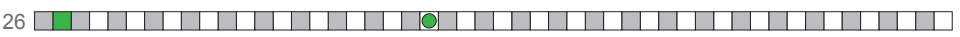

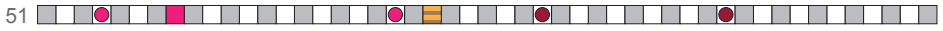

pH4.5_1_CRISPR_03

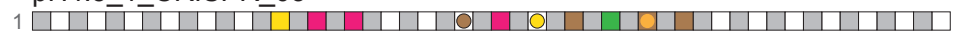

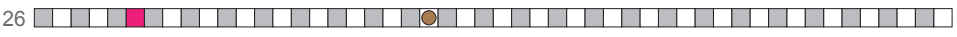

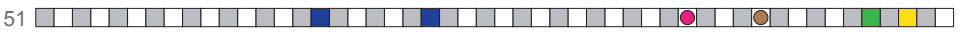

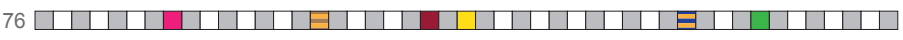

pH4.5_1_CRISPR_09

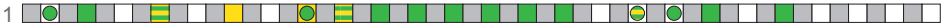

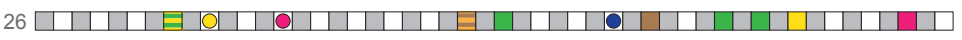

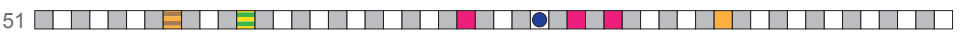

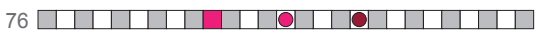

$\mathrm{pH} 4.52$ CRISPR 08

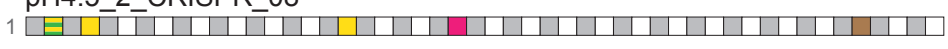
$26 \square \square$

pH4.5_2_CRISPR_09

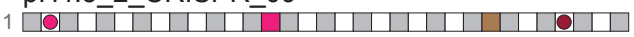

pH4.5 3 CRISPR 17

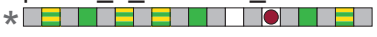

pH4.5_3_CRISPR_27

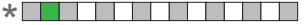

Matched spacer - 1 mismatch

560

Fig. 1. Linkage of active ${ }^{13} \mathrm{C}$-enriched viruses to Methylocystaceae populations in soil

562 by comparison of spacer sequences in CRISPR arrays. a) Distribution of spacers from 8 mVCs in Methylocystis CRISPR arrays (MAGs 5, 6 and six unbinned contigs). b)

564 CRISPR array of Methylosinus MAG 11 containing spacers linked to two mVCs. CRISPR array names describe the individual soil microcosm that the contig was 566 recovered from. DRs for complete arrays are numbered (in grey), with the spacer after DR 1 being the most recently incorporated. Two partial arrays are denoted with an *

568 Spacers with $100 \%$ identity or 1 mismatch to sequences in $\mathrm{mVCs}$ are represented by colour-coded squares and circles, respectively, with stripes representing sequences

570 found in two different mVCs. 
a)

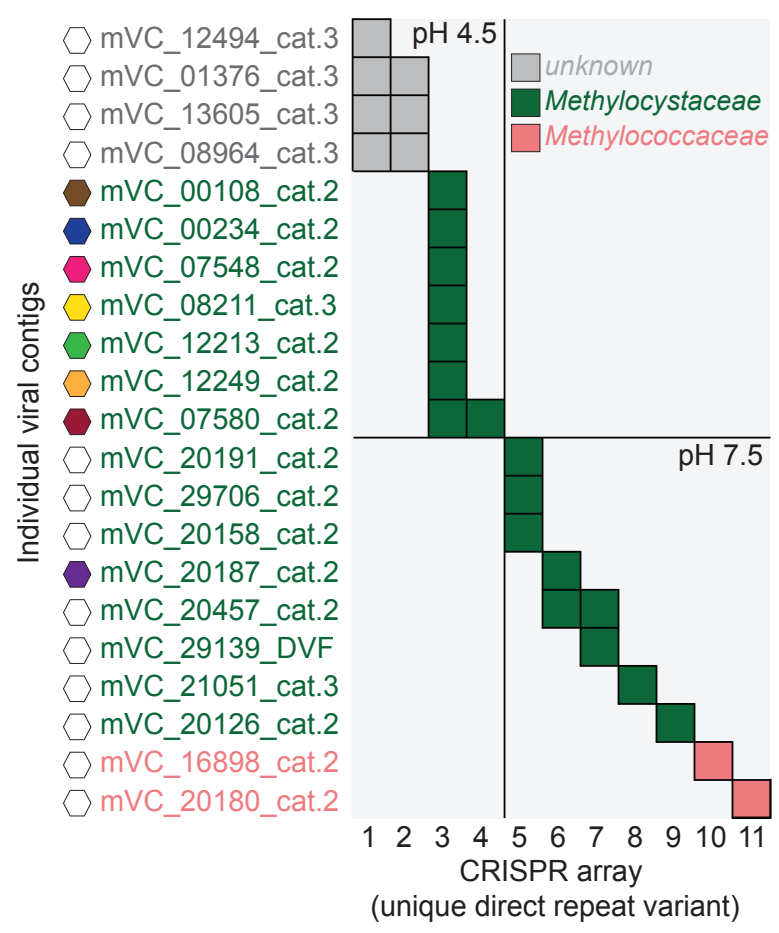

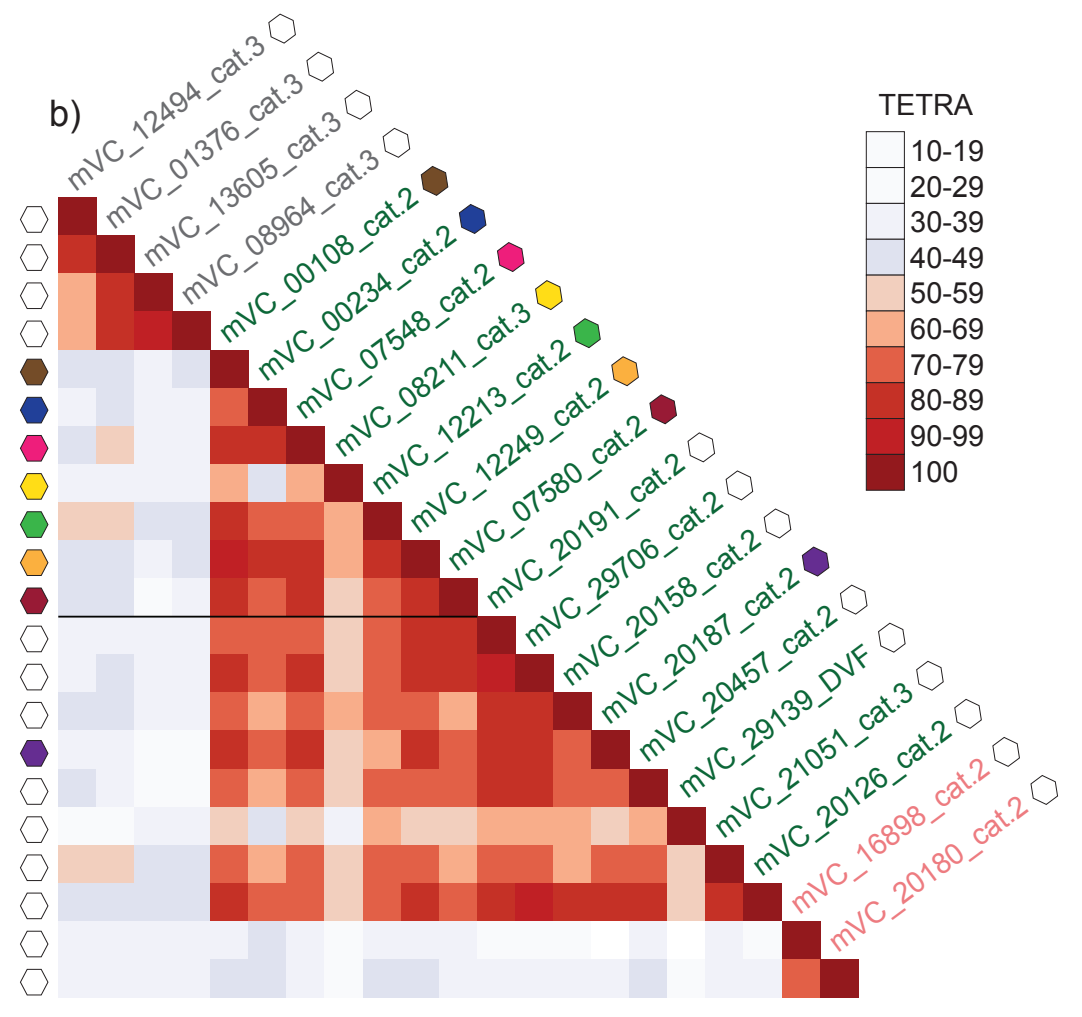

572

Fig. 2. Linkages of ${ }^{13} \mathrm{C}$-enriched viruses to CRISPR arrays in $\mathrm{pH} 4.5$ and 7.5 soil. a)

574 Presence of spacers from $21 \mathrm{mVCs}$ in 11 different CRISPR array variants (unique DR sequence). Taxonomic affiliation of CRISPR arrays to host families was determined

576 by phylogenomic analysis of affiliated MAGs $(3,6)$ or unbinned contigs $(2-5,7-9)$, or inferred from shared homologues between linked mVCs and bacterial genomes (10,

578 11). All mVCs were $>10 \mathrm{kbp}$ except mVC_08964_cat.3 (9.8 kbp) and mVC_28139_DVF (5.1 kbp). These two mVCs were also the only two predicted using

580 DeepVirFinder, with calculated probabilities describing 'likely' and 'probable' viruses, respectively. b) TETRA correlation coefficients between 21 CRISPR-linked mVCs.

582 Colour-coded hexagon symbols denote linkage to MAG-associated CRISPR arrays as per Fig. 1. 
a)

\begin{tabular}{|c|c|}
\hline Order & Family \\
\hline \multirow[t]{3}{*}{ Rhizobiales } & Methylocystaceae \\
\hline & Hyphomicrobiaceae \\
\hline & Various Rhizobia families \\
\hline Methylococcales & Methylococcaceae \\
\hline Myxococcales & Various families \\
\hline Bdellovibrionales & Bdellovibrionaceae \\
\hline
\end{tabular}

b)

Methylocystaceae viruses (80)

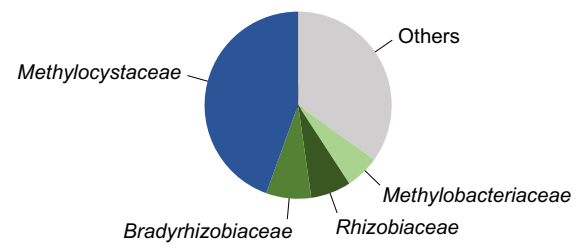

Hyphomicrobiaceae viruses (4)

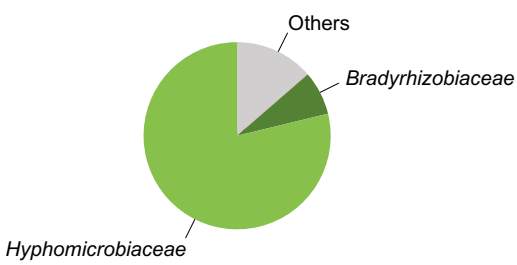

Rhizobia viruses (5)

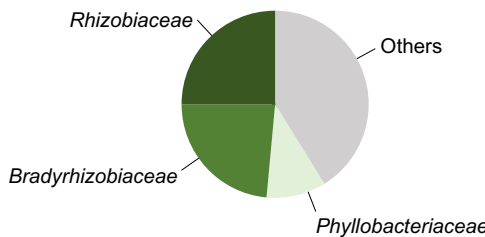

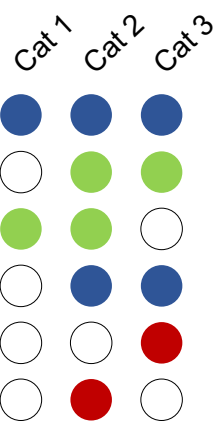

Methanotroph viruses

Non-methanotrophic methylotroph viruses

Predator viruses

Methylococcaceae viruses (10)

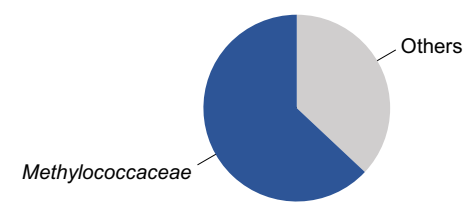

Bdellovibrionaceae viruses (1)

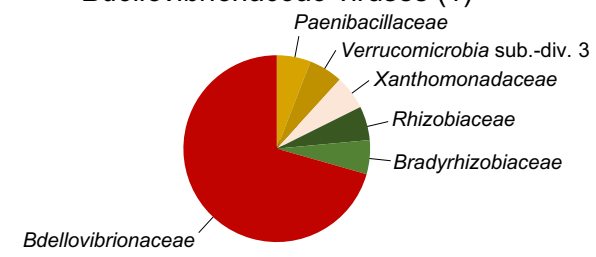

Myxococcales viruses (3)

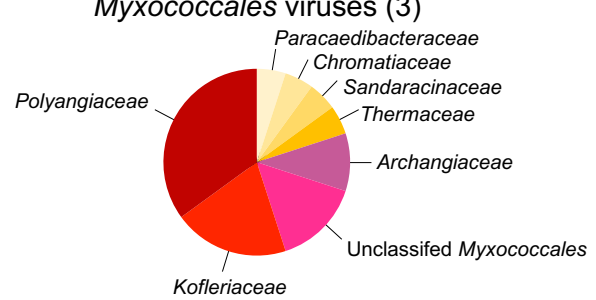

Fig. 3. Linkage of ${ }^{13} \mathrm{C}$-enriched viruses to methanotrophic, methylotrophic and predator bacterial host populations through identification of shared homologous genes. a) Association of viruses with different bacterial families

588 and functional groups inferred from the presence of $\geq 5$ shared homologous genes in category-1, -2 and -3 VirSorter-predicted mVCs. b) Proportion of homologues

590 in methanotroph, non-methanotrophic methylotroph or predator viruses linked to individual bacterial families. Each chart summarises those mVCs that all contain $\geq 5$

592 homologues to one family (number of mVCs given in parentheses) but with other taxonomic linkages also given. 'Other' describes the proportion found in families

594 each represented by less than $<5 \%$ of homologues or those not annotated to the family level. 


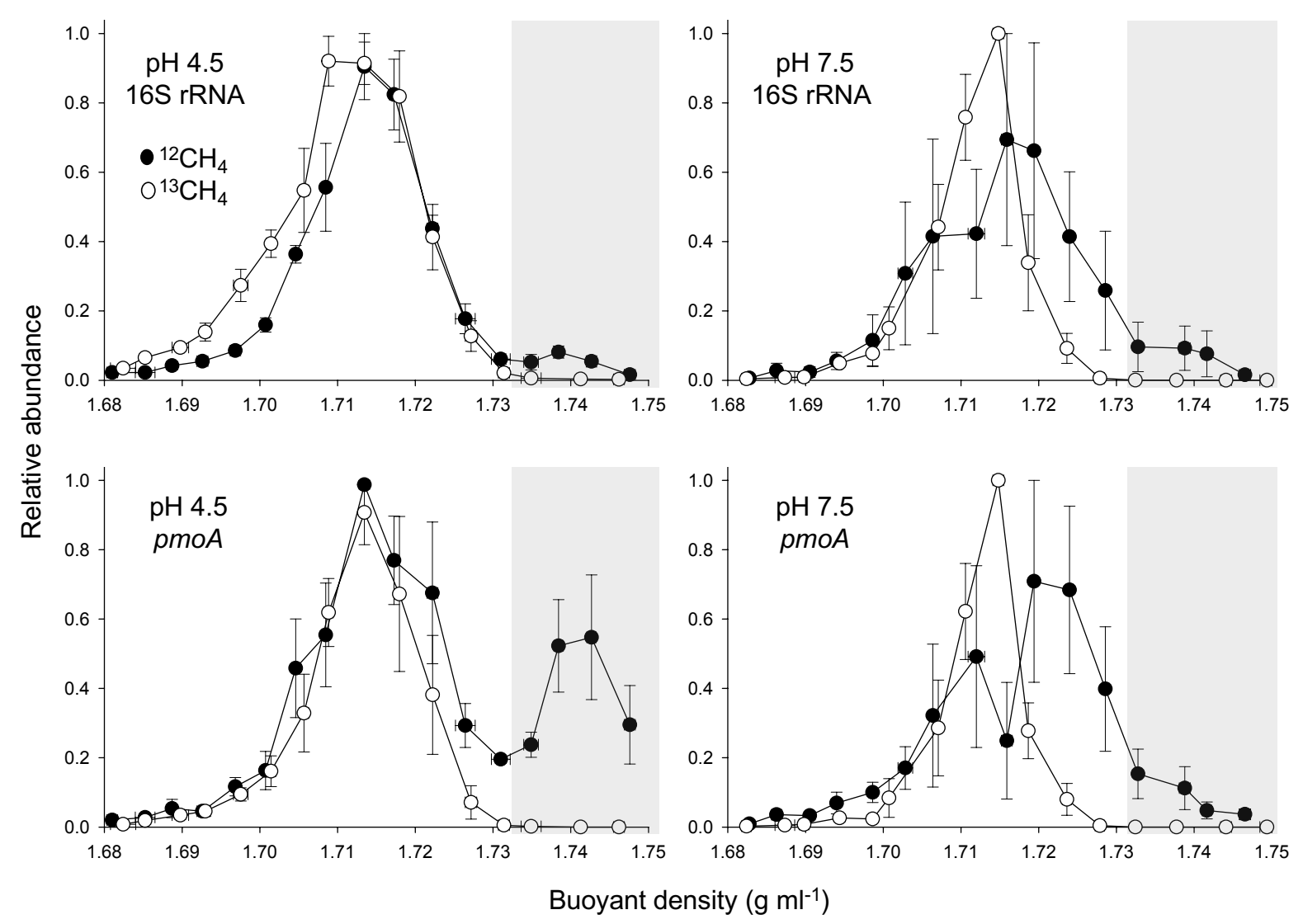

596

598

600

602 Supplementary Fig. 1. Buoyant density distribution of genomic DNA from total bacterial 16S rRNA genes and methanotroph communities possessing particulate methane monooxygenase sub-unit $\mathrm{A}(p m o A)$ genes after isopycnic centrifugation in $\mathrm{CsCl}$ gradients. Genomic DNA was extracted from triplicate $\mathrm{pH} 4.5$ and 7.5 soil 606 microcosms incubated with a $10 \%{ }^{12} \mathrm{C}$ - or ${ }^{13} \mathrm{C}-\mathrm{CH}_{4}$ headspace. Vertical error bars are the standard error of the mean relative abundance and horizontal bars (mostly smaller

608 than the symbol size) the standard error of the mean buoyant density of individual fractions from three independent $\mathrm{CsCl}$ gradients, each representing an individual 610 microcosm. The four fractions with the highest buoyant density (highlighted by grey area) were pooled for each replicate microcosm. 
a)

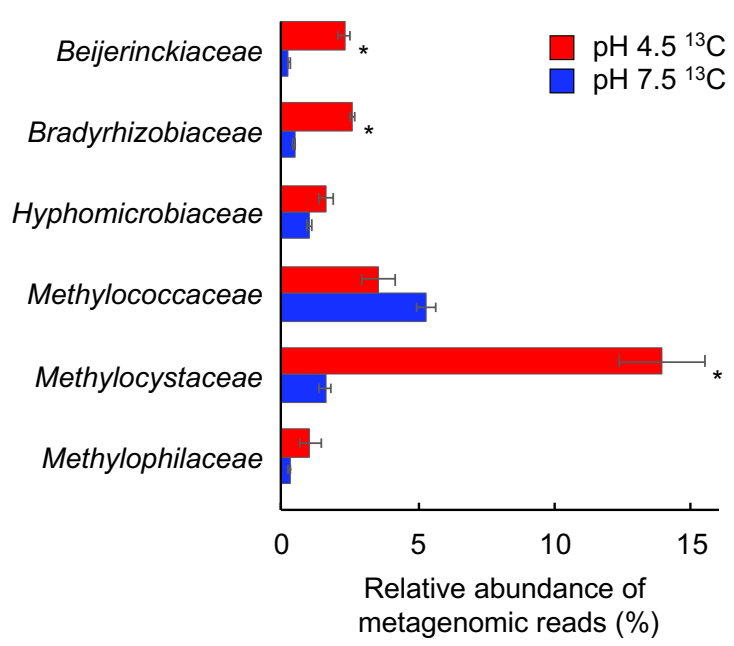

b)

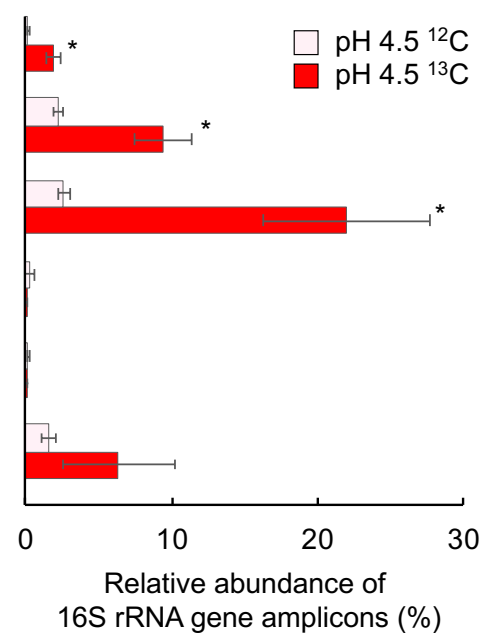

c)

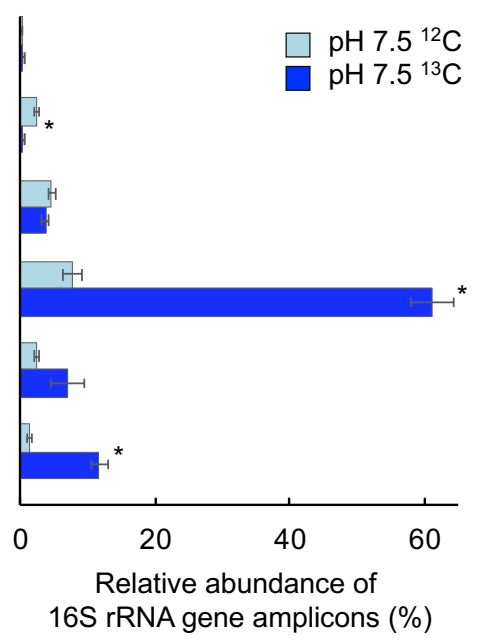

d)

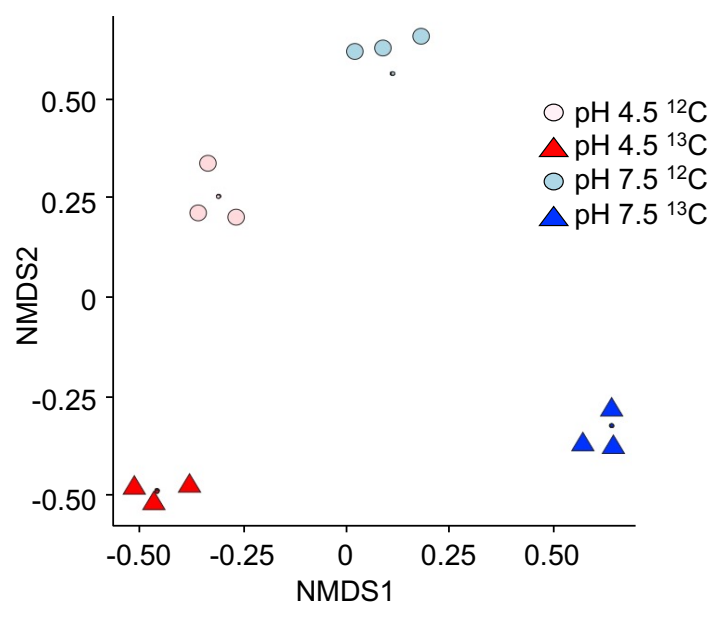

614 Supplementary Fig. 2. Taxonomic affiliation of metagenome reads and 16S rRNA gene amplified sequence variants (ASVs) derived from high buoyant density DNA from

616 triplicate $\mathrm{pH} 4.5$ and $\mathrm{pH} 7.5$ soil microcosms after incubation with ${ }^{12} \mathrm{C}$ - or ${ }^{13} \mathrm{C}_{-} \mathrm{CH}_{4}$. a) Relative abundance of metagenome sequences mapped to contigs of families that 618 recruited $\geq 1 \%$ reads in either soil. Reads were mapped to annotated contigs $\geq 5 \mathrm{kbp}$ from ${ }^{13} \mathrm{C}$-incubated microcosms only. b) and c) Relative abundance of 16S rRNA gene

620 ASVs derived from the six dominant families in ${ }^{12} \mathrm{C}-$ or ${ }^{13} \mathrm{C}_{-}-\mathrm{CH}_{4}$ incubations of $\mathrm{pH} 4.5$ and 7.5 soil, respectively. d) Non-metric multidimensional scaling of Bray-Curtis 622 dissimilarities derived from the relative abundance of annotated 16S rRNA gene ASVs. Due to the close overlap of replicates (small symbols), samples were resolved

624 for visualisation using a jitter function (large symbols). Significant differences between samples are indicated with * $(p<0.05$, two-sample Student's t-test or Welsch's t-test when variances were not homogenous). 


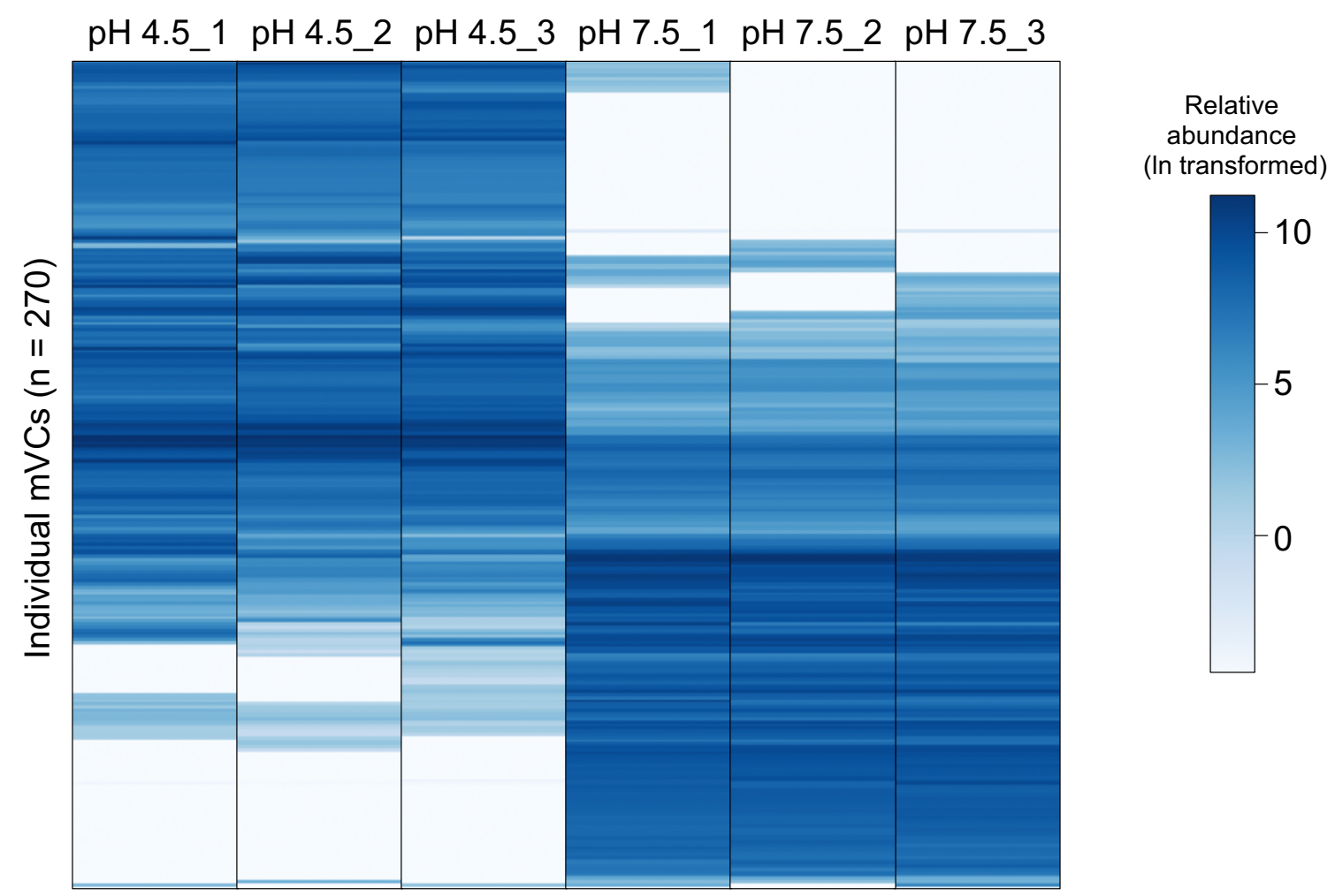

Supplementary Fig. 3. Heat-map displaying the relative abundance of $270 \mathrm{mVCs}>10$ $634 \mathrm{kbp}$ in length from ${ }^{13} \mathrm{C}$-enriched viral DNA derived from triplicate $\mathrm{pH} 4.5$ and $\mathrm{pH} 7.5$ soil microcosms. The values of normalised relative abundance are presented as reads per kbp after In transformation. 
a)

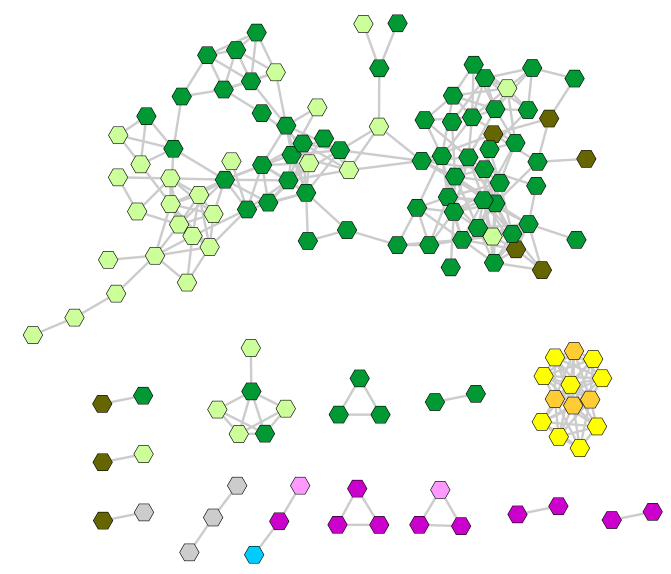

b)

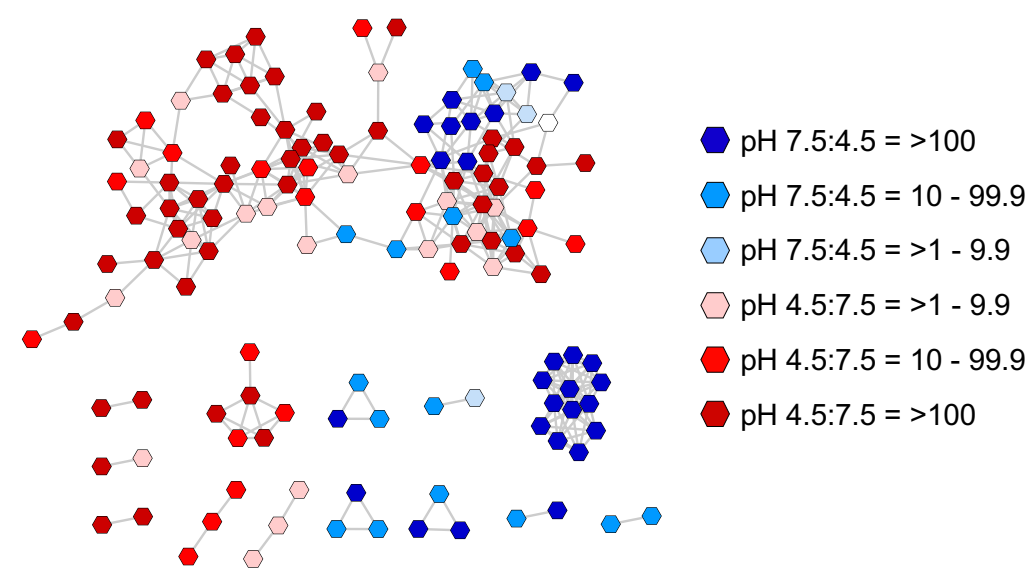

c)

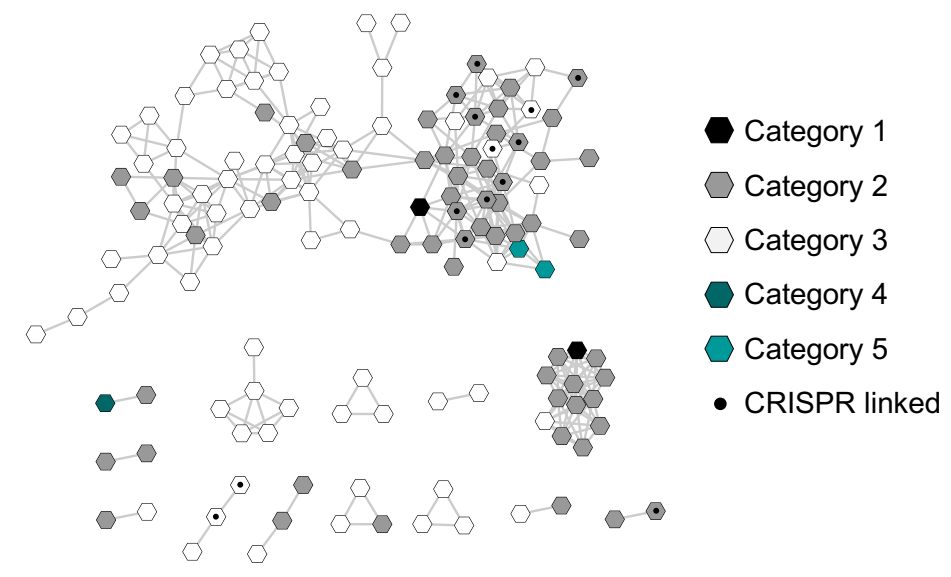

\section{Methylocystaceae \\ Methylococcaceae \\ Hyphomicrobiaceae \\ Bradyrhizobium \& Rhizobium \\ $\square$ Unknown \\ Methylocystaceae \\ from Emerson et al. (2018)}

638 Supplementary Fig. 4. Gene sharing network analysis of mVCs (one representative per vOTU) from ${ }^{13} \mathrm{C}$-enriched viruses in $\mathrm{pH} 4.5$ and 7.5 soils. a) Taxonomic affiliation

640 of hosts predicted by homologue analysis, with mVCs containing $\geq 5$ or $<5$ linked homologues highlighted. Eight mVCs from peatland soils linked to the

642 Methylocystaceae (Emerson et al., 2018) are also shown. b) Distribution of mVCs in $\mathrm{pH} 4.5$ and 7.5 soil determined by the mean ratio of normalised relative abundance

644 from triplicate samples. Seven mVCs from peatland were only found in soils with $\mathrm{pH}$ s4.7. c) VirSorter category prediction and linkage to CRISPR arrays via spacer sequence analysis. 


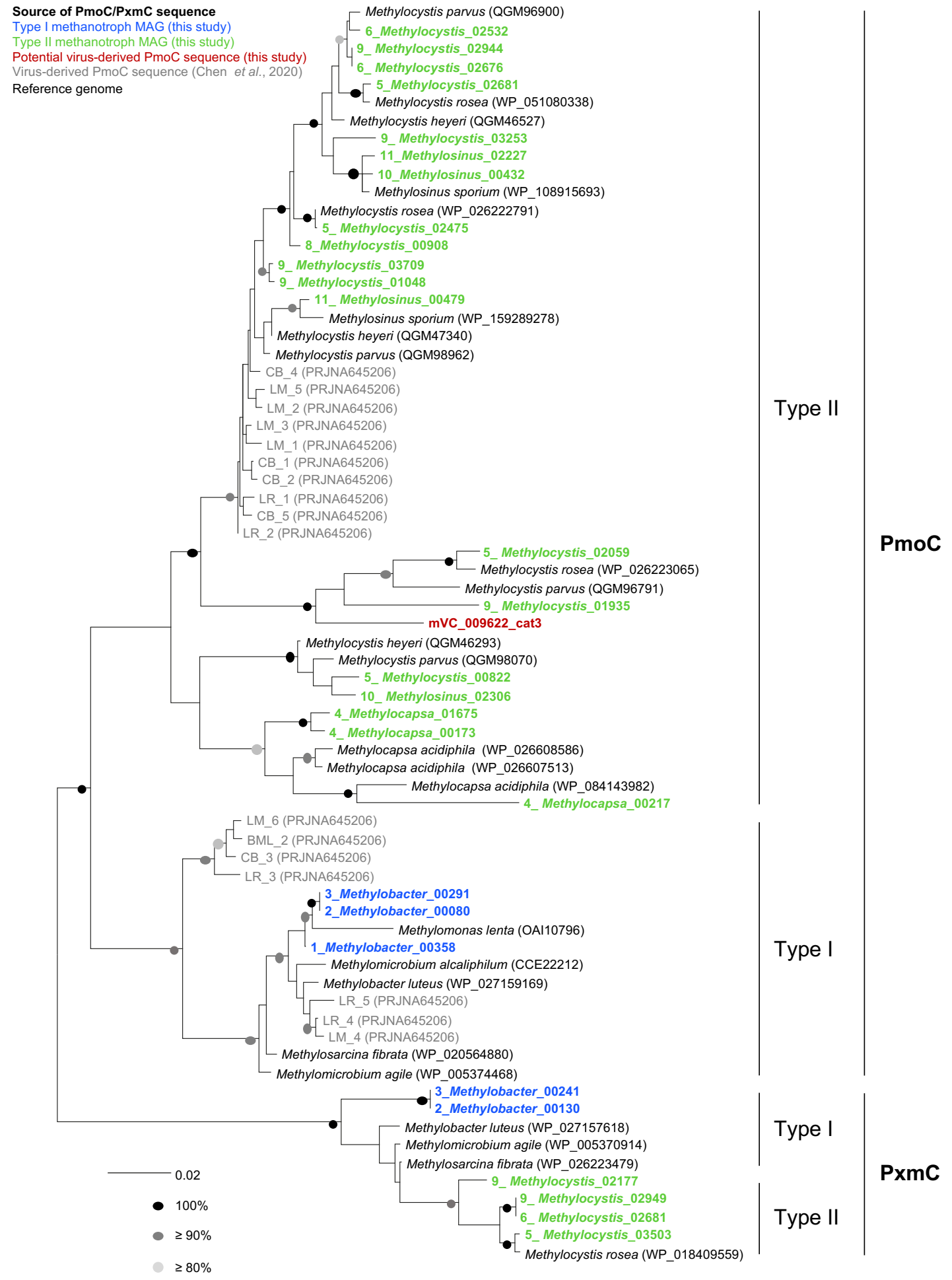

Supplementary Fig. 5. Maximum likelihood phylogenetic tree of derived amino acid sequences of PmoC and PxmC found in three type I and seven type II methanotroph MAGs and one potential viral-derived contig. MAG-derived sequences are described by MAG number, genus and contig identifier (see Supplementary Table 8). Sequences in reference methanotroph genomes and freshwater-derived viruses (Chen et al., 652 2020) were included with NCBI accession numbers given in parenthesis. Circles at nodes describe percentage bootstrap support from 100 replicates and the scale bar represents 0.02 changes per amino acid position. 


\section{Supplementary text}

656 Comparison of 16S rRNA gene amplicon and metagenomic libraries of high buoyant density DNA from ${ }^{12} \mathrm{C}$ and ${ }^{13} \mathrm{C}-\mathrm{CH}_{4}$ incubations

658 The relative abundance of annotated contigs belonging to different families in replicate metagenomic libraries was reproducible and distinct between acidic and neutral $\mathrm{pH}$

660 soils (Supplementary Figure 2a). Twenty of the 23 medium and high-quality MAGs recovered in ${ }^{13} \mathrm{C}$-derived metagenomic libraries were from methanotrophic or non-

662 methanotrophic methylotrophic populations and therefore consistent with targeting a methane-fuelled community using stable isotope probing (Supplementary Table 2).

664 Nevertheless, comparison with equivalent ${ }^{12} \mathrm{C}$ incubations was performed as per standard practice with DNA-SIP experiments ${ }^{1}$. Genomic DNA was recovered and

666 purified from high buoyant density fractions $\left(>1.732 \mathrm{~g} \mathrm{ml}^{-1}\right)$ from triplicate microcosms of both ${ }^{12} \mathrm{C}$ - and ${ }^{13} \mathrm{C}-\mathrm{CH}_{4}$ incubations. While recovered DNA from ${ }^{12} \mathrm{C}$ incubations was

668 considered too low for metagenome sequencing, with DNA concentrations below the limit of detection in some fractions, PCR amplification enabled characterisation of $16 \mathrm{~S}$

670 rRNA gene-based community structures and comparison to the equivalent fractions from ${ }^{13} \mathrm{C}$ incubations.

672 Six taxonomic families were represented by contigs to which a minimum of $\geq 1 \%$ of reads were mapped from metagenome analysis of ${ }^{13} \mathrm{C}$-enriched DNA in at least one

674 soil. These families represented $39.8 \%$ and $83.7 \%$ of $16 \mathrm{~S}$ rRNA gene ASVs in pH 4.5 and 7.5 samples, respectively, compared to 6.9 and $18.3 \%$ in the equivalent DNA

676 fractions from ${ }^{12} \mathrm{C}$-incubations (Supplementary Fig. 2). Annotated metagenome and 16S rRNA amplicon libraries generated from the same ${ }^{13} \mathrm{C}$-enriched DNA contained

678 representatives of the same C1-utilising groups, although substantial differences were observed in relative abundance. For example, while the Methylococcaceae was the

680 dominant family in $\mathrm{pH} 7.5{ }^{13} \mathrm{C}$-enriched DNA using both approaches, it represented $5.3 \%$ ( $\pm 0.3 \%$ s.e.) and $61.1 \%$ ( $\pm 3.2 \%$ s.e.) of annotated contigs and $16 \mathrm{~S}$ rRNA gene

682 ASVs, respectively. In ${ }^{13} \mathrm{C}$-enriched $\mathrm{pH} 4.5$ DNA, the dominant family in the metagenomic libraries was the Methylocystaceae, representing $13.9 \%$ ( $\pm 1.6 \%$ s.e.) of

684 annotated contigs but only $0.02 \%$ ( $\pm 0.006 \%$ s.e.) in the amplicon libraries, where the non-methanotrophic methylotrophic Hyphomicrobiaceae was the most abundant at $68622.0 \%$ ( $\pm 5.7 \%$ s.e.). Observed differences were therefore likely due to a combination of variation in genome size and 16S rRNA gene copy number between strains in 
688 addition to the range of biases associated with different marker-gene and metagenomic sequencing approaches ${ }^{2}$. Nevertheless, the overall community 690 composition determined by 16S rRNA gene amplicon libraries were highly reproducible and clearly distinct between ${ }^{12} \mathrm{C}$ and ${ }^{13} \mathrm{C}$ incubations, confirming that

692 communities analysed in metagenomic libraries were enriched in methane-derived ${ }^{13} \mathrm{C}$ (Supplementary Fig. 2c).

694

\section{Predicted C1 metabolism in metagenome assembled genomes}

696 Aerobic methylotrophic organisms utilise C1 compounds such as methane or methanol for both carbon and energy requirements ${ }^{3}$. Methanotrophs are one group of

698 methylotrophs that oxidise methane to formaldehyde via methanol, which is either assimilated for generating biomass or oxidised through to $\mathrm{CO}_{2}$ to obtain energy and

700 reductant. Non-methane oxidising methylotrophs, lacking methane monooxygenase, utilise methanol produced from other sources including that excreted from

702 methanotrophs. Methane can therefore be directly and indirectly utilised by methylotrophic populations in natural communities.

704 To determine whether MAGs represented methanotrophic or nonmethanotrophic methylotrophs, the presence of genes encoding methane

706 monooxygenase (MMO), methanol dehydrogenases (MDH) and formate dehydrogenases (FDH) was determined after predicted protein sequence annotation.

708 Taxonomic assignment and methylotrophic characterisation were consistent with known traits of Methylobacter, Methylocapsa, Methylosinus and Methylocystis strains,

710 all of which possessed particulate methane monooxygenase (pMMO) and with the three Methylosinus MAGs also possessing soluble methane monooxygenase

712 (sMMO). Eight MAGS lacked genes encoding an MMO but possessed MDH and FDH confirming methylotrophic capability. This included representatives of previously

714 recognised non-methanotrophic methylotrophs including Gemmatimonadales, Hyphomicrobium, Methylophilaceae, Methyloceanibacter plus MAGs representative of

716 the genera Rudea and Herminiimonas. One MAG belonged to the class Kiritimatiellae of the Verrucomicrobiota. While this phylum contains known methylotrophs, no

718 pathways for C1 metabolism were identified, potentially due to the low (51.5\%) estimated completeness. 
Comparison of VirSorter and DeepVirFinder in predicting virus-associated metagenome contigs

Using assembled contigs $>10 \mathrm{kbp}$, metagenomic viral contigs (mVCs) were predicted

724 using two established tools. VirSorter ${ }^{4}$ uses a database of viral genes plus analysis of virus-like motifs, and DeepVirFinder ${ }^{5}$ uses a $k$-mer based alignment-free approach,

726 using viral genomes to train the prediction model. Both approaches provide different levels of confidence. VirSorter categories 1, 2 and 3 represent 'most confident', 'likely'

728 and 'possible' virus predictions, respectively, with categories 4, 5 and 6 the equivalent for proviruses. Based on probability values, DeepVirFinder virus predictions can be 730 considered likely $(\geq 0.9, p$-value $<0.05)$ and probable $(\geq 0.7, p \text {-value }<0.05)^{6}$. We considered the matching of CRISPR array spacers with virus protospacers as the most

732 confident method of confirming a viral origin for an individual contig and facilitated comparison of the success of the two prediction tools. Of the $21 \mathrm{mVCs}$ linked using

734 CRISPR spacer analysis, 19 were predicted using VirSorter only, 1 was predicted using DeepVirFinder only and 1 predicted using both, the latter two being $<10 \mathrm{kbp}$ and

736 identified after further analysis of 5-10 kbp contigs. Of the 270 contigs predicted as category 1, 2 or 3 mVCs by VirSorter, 49 were also predicted by DeepVirFinder, which

738 uniquely identified a further 41 (of which only 4 were 'likely' viruses). These results indicate that substantially more soil virus genomes are required for training datasets

740 using an alignment-free approach.

\section{Evaluation of k-mer analysis for identifying host-virus linkages}

The matching of protospacers in mVCs to spacers in CRISPR arrays with $100 \%$

744 identity was also used to validate criteria for linkages via a 'best hit' homologue approach, specifically the sharing of a minimum of five homologues to the same

746 taxonomic family. These two approaches were then compared to linkages predicted using a $k$-mer based analysis with the tool $\mathrm{WIsH}^{7}$, which involves the comparison and

748 prediction of linkage of previously defined host- or virus-derived contigs on the basis of $k$-mer frequency analysis. Seven of the $21 \mathrm{mVCs}$ predicted to hosts via CRISPR

750 spacer analysis were linked to a host using WIsH and a probability score $\leq 0.05$ (Supplementary Table 9), all of which were consistent at the family level. Of the 103

752 mVCs linked in vConTACT 2.0 analysis with an assigned host, 35 had a host predicted using $\mathrm{WIsH}$, of which only $23(66 \%)$ and $8(23 \%)$ was the same as the homologuebased prediction at the order and family levels, respectively. These analyses therefore 
indicated that while the $k$-mer based approach using WIsH was partially successful in identifying correct linkages at the family level, it was not robust for identifying linkages with a high level of taxonomic resolution or confidence.

758

\section{Gene-sharing networks of viral metagenomic contigs}

760 Predicted hosts of mVCs from this study (using CRISPR and homologue-based approaches) were generally inconsistent with the known hosts of RefSeq viruses that were linked through vConTACT 2.0 gene-sharing network analysis ${ }^{8}$. However, mVCs from this study placed into individual clusters all had the same predicted host

764 (Supplementary Figure 4). Initial networks used mVCs with a conservative host prediction only (i.e. a minimum of $\geq 5$ homologues linked to one taxonomic family).

766 With the exception of one cluster of $3 \mathrm{mVCs}$, which were predicted to be linked to Methylococcaceae and non-methane oxidising methylotrophic Hyphomicrobiaceae,

768 all individual networks were restricted to one family for methanotrophs or one cluster of exclusively rhizobia-linked mVCs. Further analyses included all mVCs $>10 \mathrm{kbp}$ in

770 this study (i.e. including those with $<5$ host associated homologues) and the same networks were identified i.e. mVCs linked within the same cluster all had homologues

772 (i.e. with $<5$ or $\geq 5$ ) linking them to the same host family.

For Methylocystaceae mVCs, two separate but linked clusters were identified.

774 The first contained CRISPR-linked Methylocystaceae-associated mVCs, which were recovered from both $\mathrm{pH} 4.5$ and 7.5 soil (although individual mVCs were restricted to

776 one soil $\mathrm{pH}$ ). A second cluster was dominated by mVCs from $\mathrm{pH} 4.5$ soil only, indicating that most active Methylocystaceae viruses belonged to one of two distinct

778 lineages. There was a clear difference in the prediction of category-2 ('likely') and category-3 ('possible') mVCs associated with these two clusters. While category-3

780 viruses are often excluded prior to analysis of soil viromes ${ }^{9}$, CRISPR analysis demonstrated that one-third of linked mVCs were of the lowest category of confidence.

782 It must be recognised that a proportion of the predicted category-3 mVCs in this study will not be derived from viruses, and clusters composed exclusively of category-3 784 mVCs without further validation (e.g. without CRISPR spacer linkages) must be interpreted with caution. However, all major clusters identified through gene-sharing 786 network analysis contained a mixture of category-2 and -3 mVCs indicating that they represented groupings of genuine virus-derived genomes. 
An intriguing finding was the linkage of host family-specific viruses from two different geographical regions (Scotland and Sweden) and contrasting soil types

790 (agricultural loamy-sand and permafrost peatland soils), from this study and that of Emerson et al. ${ }^{9}$, respectively. In the latter study, 13 of 1,907 mVCs were predicted to

792 have a methanotroph host, of which 9 were linked specifically to the Methyocystaceae. Intriguingly, 7 of these were also linked to a predicted Methyocystaceae mVC in our

794 study, with one additional mVC predicted to have a Methylocapsa host (i.e. belonging to the Beijerinckiaceae which is another methylotrophic family of the Rhizobiales) but

also contained one predicted gene with a 'best hit' match to a Methylocystis genome homologue. As soil $\mathrm{pH}$ is recognised as one of the dominant factors driving microbial community structures in soil ${ }^{10}$, it is interesting to note that linked mVCs from both studies were also from acidic soils only.

800

\section{References}

802 1. Nicol, G.W. \& Prosser, J.I. Strategies to determine diversity, growth and activity of ammonia oxidising archaea in soil. Meth. Enzymol. 496, 3-34 (2011).

804 2. McLaren M.R., Willis, A.D. \& Callahan, B.J. Consistent and correctable bias in metagenomic sequencing experiments. eLife 8, e46923 (2019).

806 3. Chistoserdova, L., Kalyuzhnaya, M.G. \& Lidstrom, M.E. The expanding world of methylotrophic metabolism. Annu. Rev. Microbiol. 63, 477-499 (2009).

808 4. Roux, S., Enault, F., Hurwitz, B. L. \& Sullivan, M. B. VirSorter: mining viral signal from microbial genomic data. PeerJ 3, e985 (2015).

810 5. Ren, J. et al. Identifying viruses from metagenomic data by deep learning. Quant. Biol. 8, 64-77 (2020).

812 6. Trubl, G., Hyman, P., Roux, S \& Abedon, S.T. Coming-of-age characterization of soil viruses: a user's guide to virus isolation, detection within metagenomes, and $814 \quad$ viromics. Soil Syst. 4, 23 (2020).

7. Galiez, C, Siebert, M., Enault, F., Vincent, J. \& Söding, J. WisH: who is the host? Predicting prokaryotic hosts from metagenomic phage contigs. Bioinformatics 33, 3113-3114 (2017).

818 8. Jang, H.B. et al. Taxonomic assignment of uncultivated prokaryotic virus genomes is enabled by gene-sharing networks. Nat. Biotechnol. 37, 632-639 (2019).

820 9. Emerson, J.B. et al. Host-linked soil viral ecology along a permafrost thaw gradient. Nat. Microbiol. 3, 870-880 (2018). 
822 10. Bartram, A.K. et al. Exploring links between pH and bacterial community composition in soils from the Craibstone Experimental Farm. FEMS Microbiol. Ecol. 87, 403-415 (2014). 